Ghasemi et al., Afr J Tradit Complement Altern Med. (2013) 10(2):368-385 1 http://dx.doi.org/10.4314/ajtcam.v10i2.24

\title{
ETHNOBOTANICAL STUDY OF MEDICINAL PLANTS USED BY KURD TRIBE IN DEHLORAN AND ABDANAN DISTRICTS, ILAM PROVINCE, IRAN
}

\author{
*Ghasemi Pirbalouti, A., Momeni, M. and **Bahmani, M. \\ *Researches Centre of Medicinal Plants and Ethno-veterinary, Islamic Azad University, \\ Shahrekord, **Food and Beverages safety Research Center, Urmia University of Medical \\ Sciences, Urmia, Iran Branch, PO Box 166, Shahrekord, Iran \\ *E-mail: ghasemi@iaushk.ac.ir
}

\begin{abstract}
This paper provides significant ethnobotanical information on pharmaceutical plant uses, where some degree of acculturation exists, so that there is urgency in recording such data. The aim of this work is to catalogue, document, and make known the uses of plants for folk medicine in Dehloran and Abdanan districts, Ilam Province, Iran. An analysis was made of the species used, parts of the plant employed, preparation methods, administration means, and the ailments treated in relation to pathological groups. A folk botanical survey was carried out from February 2007 to October 2009. The information was collected from 81 persons (60\% men and 40\% women) in 20 villages. The informants reported data on 122 species, belonging to 49 botanical families, were claimed as medicinal. This work is focused on human medicinal plant uses, which represent $95 \%$ of the pharmaceutical uses. The most commonly represented families were Asteraceae (37.5\%), Lamiaceae (20.8\%), Rosaceae (18.7\%), Fabaceae (16.7\%) and Apiaceae (14.6\%). Some of the uses were found to be new when compared with published literature on ethnomedicine of Iran. The folk knowledge about medicinal plant use is still alive in the studied region, and a number of scarcely reported plant uses has been detected, some of them with promising phytotherapeutical applications. The results of the study reveal that some of species play an important role in primary healthcare system of these tribal communities.
\end{abstract}

Key words: Medicinal plants, ethnobotany, folklore plant, Ilam

\section{Introduction}

The Ilam province is situated on the west of Iran. This province known as "Thyme land or locally as Sarzamin-e-Avishan" and it is principally a mountainous region with plains following the direction of West Zagros range. It borders Khuzestan province in the south, Lurestan province in the east, Kermanshah province in the north and Iraq in the west with $425 \mathrm{kms}$ of common border (Figure 1). Ilam is located between latitude $31^{\circ} 58^{\prime}$ to $34^{\circ} 15^{\prime}$ $\mathrm{N}$ and longitude $45^{\circ} 24^{\prime}$ to $48^{\circ} 10^{\prime}$ E. It occupies an area of $19,086 \mathrm{~km}^{2}$. The elevation range is between $50 \mathrm{~m}$ above sea level in the south to $3060 \mathrm{~m}$ above sea level in the west province (Kabirkooh Mountain).

The climate of the region is influenced with its varied elevation. Annually, it generally rains $200 \mathrm{~mm}$ in the south and $500 \mathrm{~mm}$ in the north and the period of winter, the minimum temperature can reach $-15^{\circ} \mathrm{C}$ in north and summer is day with maximum temperature between $45^{\circ} \mathrm{C}$ in south. Humidity is generally high especially in the winter (Dec-Mar) months (Ilam Meteorology Office, 2010).

The natural vegetation is rangeland and oak forest. Flora of Ilam province is extremely rich with about 1000 species of plant (Mozaffarian, 2008). The present study focused on the current status of knowledge of folk medicine in Abdanan and Dehloran districts. These districts are situated on the west and southwest of Ilam province. The Ilam population is predominantly Feyli Kurdish. The province total population was 545,787 in 2006. The population of Abdanan and Dehloran was 47,370 and 62,256 in 2006, respectively. The northern part of the province is mostly inhabited by Kurdish tribes who speak with two dialects: Kalhuri and Feyli. The majority are Feyli Kurds, such as Kurdish tribes of Khezel, Arkawâzi, Beyrey (Ali Sherwan), Malekshahi and Shuhan. Lurs live in the southern and eastern parts of the province; for example: Abdanan, Dareh Shahr, Dehloran and Mehran. Most are Shi'a Muslims. The Kurds are traditionally nomadic people. The people's main source of living in this region is farming, agriculture, sheepherding and husbandry (MPOI, 2003).

Plants have always had an important role to play in medicine and public health. The knowledge on the use of medicinal plants was acquired by trial and error and handed on from generation to generation (Ghorbani, 2005). Nevertheless, handing down of this knowledge is in danger due to bad contacts between older and younger generations. Iran has a long medical tradition and traditional learning of medicinal plants (Ghorbani, 2005). Some authors have investigated the traditional pharmacopeia and medicinal plants in different areas of Iran (Afshar, 1990; Amin, 1991; Ayiineh Chii, 1989; Ghasemi Pirbalouti, 2009; Ghorbani, 2005; Hovayzeh et al., 2001; Miraldi et al., 2001; Mir-Heidari, 1993; Salehi Surmaghi et al., 1992; Zargari, 1989-1992). However, no information is available on the medicinal plants of the Ilam communities. In order to record all these medicinal knowledge, new or rare uses of medicinal plants and to record any use of plants in the region, the ethnobotanical survey of Ilam communities is undertaken.

The aims of this paper are:

- $\quad$ The documentation of indigenous medicinal plants used in Ilam, Iran 
Ghasemi et al., Afr J Tradit Complement Altern Med. (2013) 10(2):368-000 2 http://dx.doi.org/10.4314/ajtcam.v10i2.24

- The assessment of uses of the native species

- $\quad$ The description of the most common preparations made from herbal drugs used in Ilam ethnomedicine.

\section{Materials and Methods Plant material}

This study investigated plant material used for medicinal purposes within communities located in the Dehloran and Abdanan district, Ilam province, and involved 122 plant species. The plant specimens were collected either in the flowering or the fruiting condition, preferably both. A specimen of each species with a size of about $30 \mathrm{~cm}$ was collected. Each specimen was numbered as and when it was collected and the detailed notes were entered in the field note book. All the collected specimens were properly processed. Provisional identifications of specimens were made with the help of "Flora of Iran" (Ghahreman, 1987-1989), "Flora of Ilam" (Mozaffarian, 2008), "Encyclopedia of Iranian Plants” (Mozaffarian, 1996), Flora Iranica” (Rechinger, 1963-1998), etc. Later identifications were confirmed with the help of the authentic specimens deposited at the Herbarium of Researches Centre of Agriculture and Natural Resources of Ilam, Iran.

\section{Experimental}

An ethnobotanical survey of Abdanan and Dehloran districts, Ilam province, was conducted during February 2007 to October, 2009. The data of native medicinal plants were collected from 81 individuals comprised of herbal practitioners, young and elders (60\% men and $40 \%$ women) in 20 villages, mostly of the southern and western parts of the area. The informants were between the age of 22 to 65 years. The information was collected through questionnaire, interviews and discussions among the tribal practitioners in their local language (Kurdish). A semi-structured questionnaire was used to extract information on types of ailments treated by the use of medicinal plants and plant parts used in treating the respective ailments (Appendix 1).

\section{Data analyses}

The data collected during the fieldwork have been entered and analyzed in a database generated with Microsoft Excel 2007 (Microsoft Corporation) software. The results have been structured in a plant catalogue that considers the following items: plants mentioned (including scientific, English, Persian and local names); botanical families which these plants belong to; medicinal, edible and non edible uses separated by the part of the plant employed, and also other utilizations that can have ethnobotanical interest. Pharmaceutical methods of preparing the remedies are also part of the catalogue, as well as if the plant referred can be part of a mixture. Comparisons between the plants claimed as useful by our informants and those previously reported have been carried out consulting other previous works on ethnobotany, economic botany, medicinal plants and phytotherapy. It has been considered as very scarcely reported those not found in these papers, or appearing only in a maximum of three of them. This method has allowed us to determine the degree of originality and novelty of the uses claimed by the informants.

\section{Results \\ Medicinal plants reported}

The present study revealed that a total of 122 plants belonging to 106 genera and 49 families have been documented for their therapeutic use against different diseases (Table 1 and Figure 1). Most of the medicinal plants are collected from wild ( 93\%) and only nine species (7\%) are cultivated in an area, either in gardens or fields (Trifolium repens, Sesamum indicum, Nicotiana tabacum, Salix alba, Rosa damascena, Pimpinella anisum, Medicago sativa, Cannabis sativa and Crocus sativus). Among them 84 were herbs (68\%), 21 were shrubs (17\%) and 18 were trees (15\%) (Figure 2). The most commonly represented families were Asteraceae (37.5\%), Lamiaceae (22.92\%), Rosaceae (18.75\%), Fabaceae (16.67\%) and Apiaceae (14.58\%), Brassicaceae (10.42\%) and Chenopodiaceae (8.33\%) (Figure 1). The most abundant genus was Centaurea, three species; Allium, Amygdalus, Artemisia, Astragalus, Cerasus, Crocus, Ferula, Pisachia, Rosa, Scrophularia, Salvia, Xanthium and Ziziphus had two species (Table 1).

Plant parts used, preparation and administration

Leaves (30\%) are the most widely used plant parts even solely or mixed with other parts, followed by flowers (22\%), fruits (13\%) and stem (10\%) in some cases the whole plant (Figure 3). 


\begin{tabular}{|c|c|c|c|c|c|c|c|c|c|c|}
\hline Row & Scientific Name & Family name & $\begin{array}{c}\text { Elam Kurdish } \\
\text { name }\end{array}$ & Persian name & English name & Habit & Life cycle & Parts used & $\begin{array}{c}\text { Way of } \\
\text { application }\end{array}$ & Uses/Ailments treated \\
\hline 1 & $\begin{array}{c}\text { Achillea biebersteinii } \\
\text { Afan. }\end{array}$ & Asteraceae & $\begin{array}{c}\text { Boomaro, Berenj } \\
\text { daz, Gol Zard }\end{array}$ & $\begin{array}{l}\text { Boomadaran-e- } \\
\text { Zard }\end{array}$ & Yarrow & $\mathrm{H}$ & Perennial & Flowers, Leaves & $\begin{array}{l}\text { External/ } \\
\text { Internal }\end{array}$ & $\begin{array}{l}\text { Indigestion, rheumatism, sedative } \\
\text { (toothache), anti-septic and } \\
\text { hemagglutinate }\end{array}$ \\
\hline 2 & $\begin{array}{c}\text { Adianthum capillus-veneris } \\
\text { L. }\end{array}$ & Polypodiaceae & $\begin{array}{l}\text { Kamar Avizeh, } \\
\text { Bareh za }\end{array}$ & Parsiavoushan & $\begin{array}{l}\text { Southern } \\
\text { maidenhair, } \\
\text { Venus's hair }\end{array}$ & $\mathrm{H}$ & Perennial & Flowers, Leaves & Internal & $\begin{array}{l}\text { Anti-septic, kidney pain, anti- } \\
\text { calculus, analgesia and hair color }\end{array}$ \\
\hline 3 & Adonis dentate Delile. & Ranunculaceae & Gol Zarde & $\begin{array}{l}\text { Cheshme } \\
\text { Khorous, Gol } \\
\text { Khorousak }\end{array}$ & $\begin{array}{c}\text { Adonis, bird's } \\
\text { eye }\end{array}$ & $\mathrm{H}$ & Annual & Flower & Internal & $\begin{array}{l}\text { digestive discords and indigestion, } \\
\text { Joundice }\end{array}$ \\
\hline 4 & $\begin{array}{c}\text { Alhagi persarum Boiss. \& } \\
\text { Buhse. }\end{array}$ & Fabaceae & Agoul, Aghol & $\begin{array}{l}\text { Kharshootor, } \\
\text { Toranjabin }\end{array}$ & Camel's thorn & $\mathrm{H}$ & Perennial & Stems, Leaves & Internal & $\begin{array}{l}\text { Anti-calculus, anti-septic, kidney } \\
\text { problems, urine tube infection and } \\
\text { laxative (for baby) }\end{array}$ \\
\hline 5 & Allium akaka Gmelin. & $\begin{array}{l}\text { Aliaceae or } \\
\text { Liliaceae }\end{array}$ & $\begin{array}{l}\text { Aneshk, Anesh, } \\
\text { Valk }\end{array}$ & Valk & \begin{tabular}{|c|}
$\begin{array}{c}\text { Ramsons } \\
\text { broad, bear's } \\
\text { garlic }\end{array}$ \\
\end{tabular} & $\mathrm{H}$ & Perennial & Leaves, Bulbs & Internal & $\begin{array}{c}\text { Appetizer, anti-septic, anti-calculus, } \\
\text { anti-parasite and good digestive } \\
\text { system }\end{array}$ \\
\hline 6 & $\begin{array}{c}\text { Allium ampeloprasum L. } \\
\text { subsp. iranicum Wendelbo }\end{array}$ & $\begin{array}{l}\text { Aliaceae or } \\
\text { Liliaceae }\end{array}$ & Tareg & $\begin{array}{l}\text { Tareh Koohi, } \\
\text { Piaz Kalagh }\end{array}$ & \begin{tabular}{|c|} 
Perennial \\
sweet leek, \\
great round- \\
headed garlic
\end{tabular} & $\mathrm{H}$ & Perennial & Leaves, Bulbs & Internal & $\begin{array}{l}\text { Anti-septic, kidney infection, urine } \\
\text { infection, anti-calculus, gastric pain, } \\
\text { intestinal problem and culinary }\end{array}$ \\
\hline 7 & \begin{tabular}{|} 
Alcea angulata (Freyn \& \\
Sint) Freyn \& Sint. Ex Iljin
\end{tabular} & Malvaceae & Gole hirou & Khatmi & Mallow & $\mathrm{H}$ & Perennial & Roots & $\begin{array}{l}\text { External/ } \\
\text { Internal }\end{array}$ & $\begin{array}{l}\text { Burn, cut wound and } \\
\text { emollient }\end{array}$ \\
\hline 8 & Alyssum minus (L.) Rothm. & Brassicaceae & Ghedameh & Ghodomeh & Alyssum & $\mathrm{H}$ & Annual & Fruits & Internal & $\begin{array}{l}\text { Emollient, cough, sore throat and eyes } \\
\text { discords }\end{array}$ \\
\hline 9 & Amygdalus arabica Olivier. & Rosaceae & Bayem, Vayem & $\begin{array}{l}\text { Badam-e- } \\
\text { Koohi }\end{array}$ & Almond & $\mathrm{T}$ & Perennial & Fruits & $\begin{array}{l}\text { Internal/ } \\
\text { External }\end{array}$ & $\begin{array}{c}\text { Child ear pain, body pain (for animal) } \\
\text { and analgesic, bronchitis, anti- } \\
\text { calculus and digestive discords }\end{array}$ \\
\hline 10 & Amygdalus lycioides Spach. & Rosaceae & Taneges & Tangras & Almond & $\mathrm{T}$ & Perennial & Fruits & External & Good hair condition \\
\hline 11 & \begin{tabular}{|} 
Rhamnus pallasii Fisch. \& \\
C. A. Mey
\end{tabular} & Rhamnaceae & Arjan & Siah tangress & Buckthorn & S & Perennial & Fruits & Internal & $\begin{array}{l}\text { Cold, emollient, cough } \\
\text { and sore throat }\end{array}$ \\
\hline 12 & Anthemis altissima L. & Asteraceae & Babineh & Babooneh & Chamomile & $\mathrm{H}$ & Annual & Flower & $\begin{array}{l}\text { External/ } \\
\text { Internal }\end{array}$ & Indigestion and skin whiting \\
\hline 13 & $\begin{array}{l}\text { Aristolochia olivieri } \\
\text { Collegno in Boiss. }\end{array}$ & Aristolochiaceae & Zaravand & Chopoghak & $\begin{array}{l}\text { Dutchman's- } \\
\text { pipe }\end{array}$ & $\mathrm{H}$ & Perennial & Leaves, Stem & External & Dermal discords and wound \\
\hline
\end{tabular}




\begin{tabular}{|c|c|c|c|c|c|c|c|c|c|c|}
\hline 14 & $\begin{array}{c}\text { Calendula persica C. A. } \\
\text { Mey. }\end{array}$ & Asteraceae & Golzardeh & $\begin{array}{l}\text { Hamisheh } \\
\text { Bahar-e-Irani }\end{array}$ & $\begin{array}{l}\text { Iranian } \\
\text { marigold }\end{array}$ & $\mathrm{H}$ & Annual & Flower & External & $\begin{array}{l}\text { Dermal discords, wound } \\
\text { and eczema }\end{array}$ \\
\hline 15 & Artemisia sieberi Besser. & Asteraceae & Bookhoshkeleh & $\begin{array}{c}\text { Dermaneh-e- } \\
\text { Zagrosi }\end{array}$ & Wormwood & $\mathrm{H}$ & Annual & Stems, Leaves & Internal & $\begin{array}{l}\text { Anti-parasite, anti-diarrheal } \\
\text { and stomachic }\end{array}$ \\
\hline 16 & $\begin{array}{c}\text { Artemisia scoparia Waldst. } \\
\text { \& Kit. }\end{array}$ & Asteraceae & Salmaneh & $\begin{array}{l}\text { Jaroy-e- } \\
\text { Mashhadi }\end{array}$ & $\begin{array}{c}\text { Oriental worm } \\
\text { wood }\end{array}$ & $\mathrm{H}$ & $\begin{array}{l}\text { Annual/ } \\
\text { biennial }\end{array}$ & Inflorescence & Internal & $\begin{array}{l}\text { Indigestion, emollient } \\
\text { and sore throat }\end{array}$ \\
\hline 17 & $\begin{array}{c}\text { Astaragalus glaucacanthus } \\
\text { Fisch. }\end{array}$ & Fabaceae & $\begin{array}{l}\text { Miveh } \\
\text { badkonaki }\end{array}$ & Asbi gavan & Astragal & $S$ & Perennial & Fruits & Internal & $\begin{array}{c}\text { Used in food and confectionery, tonic, } \\
\text { gastric pain, headache } \\
\text { and wild fruit }\end{array}$ \\
\hline 18 & $\begin{array}{l}\text { Astragalus gossypinus } \\
\text { Fisch. }\end{array}$ & Fabaceae & Gavan & $\begin{array}{l}\text { Gavan-e- } \\
\text { panbehi }\end{array}$ & Astragal & $\mathrm{S}$ & Perennial & Resin & $\begin{array}{c}\text { External/ } \\
\text { Internal } \\
\end{array}$ & $\begin{array}{c}\text { Good hair condition } \\
\text { and cold }\end{array}$ \\
\hline 19 & $\begin{array}{c}\text { Cotoneaster lurestanica } \\
\text { Klotz. }\end{array}$ & Rosaceae & Shir khesht & $\begin{array}{l}\text { Shirkhesht-e- } \\
\text { lorestani }\end{array}$ & Cotoneaster & $\mathrm{S}$ & Perennial & Resin & Internal & Laxative for baby \\
\hline 20 & $\begin{array}{c}\text { Atriplex leucoclada (Boiss.) } \\
\text { Aellen. }\end{array}$ & Chenopodiaceae & Ramt & $\begin{array}{c}\text { Salmaki saghe } \\
\text { safid }\end{array}$ & Saltbush & $\mathrm{H}$ & Perennial & Leaves & Internal & $\begin{array}{l}\text { Emollient, cough and } \\
\text { sore throat }\end{array}$ \\
\hline 21 & Avena wiestii Steud. & Poaceae & Ganem giah & Youlaf & Wild oat & $\mathrm{H}$ & Annual & Seed & Internal & $\begin{array}{c}\text { Gastric pain, indigestion, rheumatism } \\
\text { and tonic }\end{array}$ \\
\hline 22 & Cannabis sativa L. & Canabinaceae & Shadone & Shahdoneh & Hemp & $\mathrm{H}$ & Annual & Fruits & Internal & $\begin{array}{l}\text { Seed use for nut, laxative, anti- } \\
\text { parasite, tonic, cough and nerve } \\
\text { system discords }\end{array}$ \\
\hline 23 & Capparis spinosa L. & Capparidaceae & $\begin{array}{c}\text { Kelkam, } \\
\text { Shafileh, Shafalk }\end{array}$ & Kavar & Caper & $\mathrm{H} / \mathrm{S}$ & Perennial & $\begin{array}{l}\text { Leaves, Roots, } \\
\text { Bark and Fruits }\end{array}$ & Internal & $\begin{array}{l}\text { Root bark: hepato-protective, Stem } \\
\text { bark: toothache, Leaves and } \\
\text { Fruits: Regulation of blood sugar }\end{array}$ \\
\hline 24 & Cardaria draba (L.) Desv. & Brassicaceae & Tof veh sereh & Ozmak & Hoary cress & $\mathrm{H}$ & Perennial & Young leaves & Internal & Culinary and tonic \\
\hline 25 & Carthamus oxyacantha M.В. & Asteraceae & $\begin{array}{l}\text { Khar kharon, } \\
\text { Zardeh siri, } \\
\text { Zardeh drag }\end{array}$ & $\begin{array}{l}\text { Golerang-e- } \\
\quad \text { zard }\end{array}$ & Safflower & $\mathrm{H}$ & Annual & Flowers & Internal & $\begin{array}{l}\text { For women period discords } \\
\text { and menorrhagia }\end{array}$ \\
\hline 26 & $\begin{array}{c}\text { Centaurea iberica Trev. Ex } \\
\text { Spreng. }\end{array}$ & Asteraceae & Asan darag & $\begin{array}{l}\text { Gole Gandom- } \\
\text { e-chaman zar }\end{array}$ & Centaurea & $\mathrm{H}$ & Annual & Flowers & Internal & Gastric pain \\
\hline 27 & Centaurea intricate Boiss. & Asteraceae & Benjek dargi & $\begin{array}{l}\text { Gole Gandom- } \\
\text { e-darham } \\
\text { barham }\end{array}$ & Centaurea & $\mathrm{H}$ & Perennial & Flowers & Internal & Indigestion and gastric pain \\
\hline 28 & $\begin{array}{l}\text { Centaurea ovina Pall. Ex } \\
\text { Willd. }\end{array}$ & Asteraceae & Tilage & Gole Gandom & Centaurea & $\mathrm{H}$ & Annual & Flowers & Internal & Indigestion and gastric pain \\
\hline 29 & $\begin{array}{l}\text { Cerasus mahaleb (L.) } \\
\text { Miller. }\end{array}$ & Rosaceae & $\begin{array}{c}\text { Beralik, } \\
\text { Heloneh, } \\
\text { Mahloo }\end{array}$ & Mahlab & $\begin{array}{l}\text { Mahaleb } \\
\text { cherry }\end{array}$ & $\mathrm{T}$ & Perennial & Fruits & Internal & $\begin{array}{c}\text { Laxative, anti-calculus, culinary and } \\
\text { spice and } \\
\text { wild fruit: stomachic }\end{array}$ \\
\hline 30 & Cerasus microcarpa (C.A. & Rosaceae & Beralik, Helaneh & Albaloy-e- & Sour cherry & $\mathrm{T}$ & Perennial & Bark, Resin & Internal & Sedative, anti-calculus \\
\hline
\end{tabular}




\begin{tabular}{|c|c|c|c|c|c|c|c|c|c|c|}
\hline & $\begin{array}{l}\text { Mey) Boiss. subsp. } \\
\text { microcarpa }\end{array}$ & & & vahshi & & & & & & and anti-fever \\
\hline 31 & Cichorium intybus L. & Asteraceae & Kasni & Kasni & Chicory & $\mathrm{H}$ & Perennial & $\begin{array}{l}\text { Root, Stem, } \\
\text { Leaves }\end{array}$ & $\begin{array}{l}\text { Internal/ } \\
\text { External }\end{array}$ & $\begin{array}{l}\text { Laxative, diuretic, gastric pain and } \\
\text { dermal discords }\end{array}$ \\
\hline 32 & $\begin{array}{l}\text { Cirsium congestum Fisch. \& } \\
\text { C. A. mey. Ex DC. }\end{array}$ & Asteraceae & Kangar darag & Kagar-e-anboh & Bull thistle & $\mathrm{H}$ & Biannual & Stem & Internal & Anti-septic for gastric \\
\hline 33 & $\begin{array}{l}\text { Citrullus colocynthis (L.) } \\
\text { Schrad. }\end{array}$ & Cucurbitaceae & $\begin{array}{c}\text { Shoomi } \\
\text { sheytoneh, } \\
\text { Shomi tilaneh }\end{array}$ & $\begin{array}{l}\text { Hanzal, } \\
\text { Hendevaneh } \\
\text { Abougahl } \\
\end{array}$ & Citrul/Citrule & $\mathrm{H}$ & Perennial & Fruits & $\begin{array}{l}\text { External/ } \\
\text { Internal }\end{array}$ & Diabetes and wound \\
\hline 34 & Colchicum kotschyi Boiss. & Iridaceae & Kirgeh keh ran & Gol-e-hasrat & $\begin{array}{l}\text { Autumn } \\
\text { saffron }\end{array}$ & $\mathrm{H}$ & Perennial & Flowers & External & Rheumatism \\
\hline 35 & Crataegus pontica C. Koch. & Rosaceae & Gich & Zalzalak & Azarole & $\mathrm{T}$ & Perennial & Fruits, Leaves & Internal & $\begin{array}{l}\text { Edible as wild fruit, heart tonic, } \\
\text { antihypertensive } \\
\text { and headache }\end{array}$ \\
\hline 36 & Crocus haussknechtii Boiss. & Iridaceae & Pishog & Joo ghasem & Crocus & $\mathrm{H}$ & Perennial & Flowers & Internal & $\begin{array}{c}\text { Anti-septic for gastric } \\
\text { and stomachic }\end{array}$ \\
\hline 37 & Crocus sativus L. & Iridaceae & Kal mas & Zaferan & Saffron & $\mathrm{H}$ & Perennial & Stigma, Style & Internal & $\begin{array}{l}\text { Breezy, tonic for heart and culinary } \\
\text { use }\end{array}$ \\
\hline 38 & Daphne mucronata Royle. & Thymelaeaceae & Toye, alef & Khoshak & Daphne & $\mathrm{S}$ & Perennial & Wood & External & Cleaning eyes and eye pain (Surmeh) \\
\hline 39 & Datura innoxia Miller. & Solanaceae & Tatureh & $\begin{array}{l}\text { Datureh-e- } \\
\text { goldorosht }\end{array}$ & Hindu datura & $\mathrm{H}$ & Annual & Latex & External & Anti-wart \\
\hline 40 & $\begin{array}{l}\text { Consolida orientalis (Gey) } \\
\text { Schrood. }\end{array}$ & Ranunculaceae & Zaban ghafa & $\begin{array}{c}\text { Zaban pas } \\
\text { ghafa-e-denae }\end{array}$ & Larkspur & $\mathrm{H}$ & Annual & Flowers & Internal & Laxative and anti-parasite \\
\hline 41 & Dianthus orientalis Adam. & Caryophylaceae & Gole Mikhak & Mikhak & Pink & $\mathrm{H}$ & Perennial & Flowers, Fruits & $\begin{array}{c}\text { External/ } \\
\text { Internal }\end{array}$ & Toothache and anti-spasm \\
\hline 42 & Echinops viscidulus Mozaff. & Asteraceae & Ghane shakrook & Shekar Tighal & Globe thistle & $\mathrm{H}$ & Perennial & Bulb & Internal & $\begin{array}{l}\text { Cough, cold, sore throat and edible as } \\
\text { vegetable }\end{array}$ \\
\hline 43 & Echium italicum L. & Boraginaceae & Gole gazou & Gavzaban & $\begin{array}{l}\text { Viper's } \\
\text { bugloss }\end{array}$ & $\mathrm{H}$ & Biannual & Flower & Internal & $\begin{array}{l}\text { Nervous system relaxant, carminative, } \\
\text { cold, sore throat, wound and soporific }\end{array}$ \\
\hline 44 & Elaeagnus angustifolia L. & Elaeagnaceae & Ardegon & $\begin{array}{c}\text { Sanjed, } \\
\text { Pestanak } \\
\end{array}$ & Oleaster & $\mathrm{T}$ & Perennial & Fruits & Internal & $\begin{array}{c}\text { Anti-diarrheal, gastric pain } \\
\text { and hepatoprotective }\end{array}$ \\
\hline 45 & $\begin{array}{c}\text { Ephedra ciliata } \\
\text { Fisch. ex C. A. Mey }\end{array}$ & Ephedraceae & $\begin{array}{l}\text { Rish boz, Kori } \\
\text { feri }\end{array}$ & $\begin{array}{c}\text { Ormak, rish } \\
\text { boz }\end{array}$ & Joint fir & $\mathrm{S}$ & Perennial & Root, Stem & Internal & Anti-bacterial and anti-fever \\
\hline 46 & $\begin{array}{c}\text { Euphorbia macroclada } \\
\text { Boiss. }\end{array}$ & Ephurbiaceae & Shirghoteghan & Farfiun & Milkwort & $\mathrm{H}$ & Perennial & Latex & External & Anti-wart \\
\hline 47 & $\begin{array}{l}\text { Ferula haussknechtii } \\
\text { Wolff ex Rech. f. }\end{array}$ & Apiaceae & Komeh, Komieh & Koma & Giant fennel & $\mathrm{H}$ & Perennial & $\begin{array}{l}\text { Stem, Leaves, } \\
\text { Inflorescence }\end{array}$ & External & Anti-septic (smoking) \\
\hline
\end{tabular}




\begin{tabular}{|c|c|c|c|c|c|c|c|c|c|c|}
\hline 48 & $\begin{array}{l}\text { Ferula behboudiana } \\
\text { (Rech. f. \& Esfand) } \\
\text { Chamberlain. }\end{array}$ & Apiaceae & Anio & $\begin{array}{l}\text { Koma-e- } \\
\text { lorestani }\end{array}$ & Giant fennel & $\mathrm{H}$ & Perennial & $\begin{array}{l}\text { Stem, Leaves, } \\
\text { Inflorescence }\end{array}$ & External & Anti-septic (smoking) \\
\hline 49 & $\begin{array}{l}\text { Ferulago angulata } \\
\text { (Schlecht.) Boiss. }\end{array}$ & Apiaceae & Chavir & $\begin{array}{l}\text { Chavil-e- } \\
\text { shevidi }\end{array}$ & Ferulago & $\mathrm{H}$ & Perennial & Leaves & Internal & Anti-septic, spice and air fresher \\
\hline 50 & Ficus carica L. & Moraceae & Anjir & Anjir & Fig & $\mathrm{T}$ & Perennial & Fruits, Latex & $\begin{array}{l}\text { Internal/ } \\
\text { External }\end{array}$ & Laxative, cough and anti-wart \\
\hline 51 & Fritillaria imperialis L. & Liliaceae & Sosan gol & $\begin{array}{c}\text { Ashke } \\
\text { maryam, } \\
\text { Laleh vajhgon } \\
\end{array}$ & $\begin{array}{l}\text { Crown } \\
\text { imperial }\end{array}$ & $\mathrm{H}$ & Perennial & Bulb & External & Rheumatism and sciatica \\
\hline 52 & Fumaria parviflora Lam. & Fumariaceae & Shatareh & $\begin{array}{l}\text { Shahtareh-e- } \\
\text { irani }\end{array}$ & Fumitory & $\mathrm{H}$ & Annual & \begin{tabular}{|c|}
$\begin{array}{c}\text { Flowers, Stem, } \\
\text { Leaves }\end{array}$ \\
\end{tabular} & External & $\begin{array}{c}\text { Dermal discords, wound } \\
\text { and eczema }\end{array}$ \\
\hline 53 & $\begin{array}{l}\text { Glycyrrhiza glabra L. var. } \\
\text { glabra }\end{array}$ & Fabaceae & Balik & Shirin bayan & Licorice & $\mathrm{H}$ & Perennial & Roots, Flowers & Internal & $\begin{array}{l}\text { Gastric ulcer, digestive discords, } \\
\text { duodenal pain, stomach stranger, } \\
\text { diabetes, intestinal pain and culinary }\end{array}$ \\
\hline 54 & Gundelia tournefortii L. & Asteraceae & Kenyer & Kangar & - & $\mathrm{H}$ & Perennial & Leaves, Stem & Internal & $\begin{array}{c}\text { Edible as vegetable, indigestion, } \\
\text { tonic, laxative, } \\
\text { anti-calculus, diabetes and culinary }\end{array}$ \\
\hline 55 & Hypericum scabrum L. & Hypericaceae & Siveh ran & Gol-e-raye & St. John's wort & $\mathrm{H}$ & Perennial & Inflorescence & Internal & $\begin{array}{l}\text { Green tea, sedative, headache and } \\
\text { nerve system relaxant }\end{array}$ \\
\hline 56 & Isatis raphanifolia Boiss. & Brassicaceae & Vasmeh & Vasmeh & Dyer's woad & $\mathrm{H}$ & Annual & $\begin{array}{l}\text { Root, Leaves, } \\
\text { Seed }\end{array}$ & External & Coloring for hair \\
\hline 57 & $\begin{array}{l}\text { Lonicera nummulariifolia } \\
\text { Jaub. \& Spach. }\end{array}$ & Caprifoliaceae & Pela khor, Shan & Pelakhor & Lonicera & $\mathrm{S}$ & Perennial & Leaves, Flowers & Internal & $\begin{array}{l}\text { Anti-fever, anti-diarrheal, sedative } \\
\text { and cough }\end{array}$ \\
\hline 58 & Lycium depressum Stocks. & Solanaceae & Khoshk & Gorg tigh & Wolf berry & $\mathrm{S}$ & Perennial & Leaves, Fruits & $\begin{array}{l}\text { Internal/ } \\
\text { External }\end{array}$ & Kidney problems \\
\hline 59 & Malva neglecta Wallr. & Malvaceae & Toli, Tole & Panirak & Mallow & $\mathrm{H}$ & $\begin{array}{l}\text { Annual, } \\
\text { biennial }\end{array}$ & Leaves, Flowers & Internal & $\begin{array}{c}\text { Interstitial infection, laxative, sore } \\
\text { throat and asthma }\end{array}$ \\
\hline 60 & Medicago sativa L. & Fabaceae & Vinjeh & Younjeh & alfalfa & $\mathrm{H}$ & Perennial & Leaves, Flowers & Internal & Tonic and fattening \\
\hline 61 & $\begin{array}{l}\text { Mentha longifolia (L.) } \\
\text { Hudson. }\end{array}$ & Lamiaceae & Pineh & $\begin{array}{l}\text { Pooneh, } \\
\text { Podneh }\end{array}$ & Horsemint & $\mathrm{H}$ & Perennial & Leaves, Flowers & Internal & $\begin{array}{l}\text { Carminative, edible as vegetable and } \\
\text { flavoring }\end{array}$ \\
\hline 62 & Myrtus communis L. & Myrtaceae & Moort & Mord & Myrtle & $\mathrm{T}$ & Perennial & Leaves & $\begin{array}{l}\text { External/ } \\
\text { Internal }\end{array}$ & $\begin{array}{l}\text { Anti-septic (smoking), women } \\
\text { diseases, wound (antimicrobial) } \\
\text { and air freshener }\end{array}$ \\
\hline
\end{tabular}




\begin{tabular}{|c|c|c|c|c|c|c|c|c|c|c|}
\hline 63 & Narcissus tazetta L. & Amaryllidaceae & Gole Narges & Narges & $\begin{array}{c}\text { Polyanthus } \\
\text { narcissus }\end{array}$ & $\mathrm{H}$ & Perennial & $\begin{array}{l}\text { Flowers and } \\
\text { Bulb }\end{array}$ & $\begin{array}{l}\text { Internal/ } \\
\text { External }\end{array}$ & $\begin{array}{c}\text { Flowers: Aromatic or aromatherapy } \\
\text { (sedative, headache and cold), anti- } \\
\text { parasite and abortion }\end{array}$ \\
\hline 64 & $\begin{array}{l}\text { Nasturtium officinale } \\
\text { (L.) R. Br. }\end{array}$ & Brassicaceae & $\begin{array}{l}\text { Koleh shak, } \\
\text { Balmak }\end{array}$ & $\begin{array}{l}\text { Alaf-e- } \\
\text { cheshmeh }\end{array}$ & Water cress & $\mathrm{H}$ & Perennial & $\begin{array}{l}\text { Leaves, Flowers, } \\
\text { Roots }\end{array}$ & Internal & Stomachic, anti-parasite \\
\hline 65 & Nepeta persica Boiss. & Lamiaceae & Poneh say & $\begin{array}{l}\text { Poneh say-e- } \\
\text { Irani }\end{array}$ & Catmint & $\mathrm{H}$ & Perennial & Leaves,Flowers & $\begin{array}{l}\text { Internal/ } \\
\text { External }\end{array}$ & Carminative and anti-urticarial \\
\hline 66 & Nerium oleander L. & Apocynaceae & $\begin{array}{l}\text { Hortil, Jeleh, } \\
\text { Gharjalak }\end{array}$ & $\begin{array}{l}\text { Kharzahreh, } \\
\text { Kish }\end{array}$ & Oleander & $\mathrm{S}$ & Perennial & $\begin{array}{c}\text { Leaves, Flowers, } \\
\text { Latex }\end{array}$ & $\begin{array}{l}\text { External/ } \\
\text { Internal }\end{array}$ & $\begin{array}{l}\text { External: Burn, wound healing and } \\
\text { eczema, Internal: diuretic and heart } \\
\text { tonic }\end{array}$ \\
\hline 67 & Nicotiana tabacum L. & Solanaceae & Tanbakoo & Toton & Tobacco & $\mathrm{H}$ & Annual & Leaves & External & $\begin{array}{l}\text { Anti-leech (Limnatis nilotica), } \\
\text { anti-dermatophytosis and used } \\
\text { veterinary (health animal) }\end{array}$ \\
\hline 68 & $\begin{array}{c}\text { Noaea mucronata (Forssk.) } \\
\text { Asch \&Schweinf. }\end{array}$ & Chenopodiaceae & Khargo & $\begin{array}{l}\text { Nakhon-e- } \\
\text { aroos }\end{array}$ & - & $\mathrm{S}$ & Perennial & Leaves, Flowers & Internal & Anti-calculus \\
\hline 69 & $\begin{array}{c}\text { Onobrychis elymaitica } \\
\text { Boiss. \& Hausskn. ex } \\
\text { Boiss. }\end{array}$ & Fabaceae & Pieh kol & Speres-e-elami & Sainfoin & $\mathrm{H}$ & Perennial & Leaves, Flowers & Internal & Anti-calculus, kidney problems \\
\hline 70 & $\begin{array}{c}\text { Opoponex hispidus (Friv.) } \\
\text { Griseb. }\end{array}$ & Apiaceae & Alaf shir & Koma & Opopnax & $\mathrm{H}$ & Annual & $\begin{array}{l}\text { Stem, Leaves, } \\
\text { Inflorescence }\end{array}$ & External & Anti-septic (smoking) \\
\hline 71 & $\begin{array}{l}\text { Paliurus spina-christi } \\
\text { Miller. }\end{array}$ & Rhamnaceae & Dereg dar & Siyah telo & Christ's thorn & $\mathrm{S}$ & Perennial & Fruits & Internal & $\begin{array}{l}\text { Anti-hypertensive and } \\
\text { reduced cholesterol }\end{array}$ \\
\hline 72 & Papaver dubium L. & Papaveraceae & Gole soreh & $\begin{array}{l}\text { Khashkash-e- } \\
\text { tannaz }\end{array}$ & $\begin{array}{c}\text { Great scarlet } \\
\text { poppy }\end{array}$ & $\mathrm{H}$ & Annual & Leaves, Flowers & Internal & $\begin{array}{l}\text { Nerve system relaxant, } \\
\text { sedative }\end{array}$ \\
\hline 73 & Peganum harmala L. & Zygophyllaceae & Span & Spand, Sphand & $\begin{array}{c}\text { Harmel } \\
\text { peganum }\end{array}$ & $\mathrm{H}$ & Perennial & Fruits, Seeds & External & $\begin{array}{l}\text { Anti-septic for air, scorpion bite, } \\
\text { snake bite, toothache } \\
\text { and soporific }\end{array}$ \\
\hline 74 & Periploca aphylla Decne. & Asclepiadaceae & Kholf & Gishder & Silk vine & $\mathrm{S}$ & Perennial & Leaves, Flowers & External & Anti-inflammatory \\
\hline 75 & Phlomis olivieri Benth. & Lamiaceae & $\begin{array}{l}\text { Labeh goshak, } \\
\text { Giveh balkeh }\end{array}$ & $\begin{array}{l}\text { Goshbareh, } \\
\text { Bareh Gosh, } \\
\text { Chalmah } \\
\end{array}$ & Phlomis & $\mathrm{H}$ & Perennial & Leaves, Flowers & Internal & Carminative \\
\hline 76 & Physalis divaricata D. Don. & Solanaceae & $\begin{array}{c}\text { Arosak postheh } \\
\text { pardeh }\end{array}$ & $\begin{array}{c}\text { Arosak postheh } \\
\text { pardeh }\end{array}$ & Winter cherry & $\mathrm{H}$ & Annual & Fruits & Internal & Kidney discords \\
\hline 77 & Picnomon acarna (L.) Cass. & Asteraceae & Gemal diom & Zard khar & $\begin{array}{c}\text { Yellow plume } \\
\text { thistle }\end{array}$ & $\mathrm{H}$ & Annual & Leaves & Internal & $\begin{array}{l}\text { Indigestion, gastric discords } \\
\text { and stomachic }\end{array}$ \\
\hline
\end{tabular}




\begin{tabular}{|c|c|c|c|c|c|c|c|c|c|c|}
\hline 78 & Pimpinella anisum L. & Apiaceae & Vaveh shing & Badian romi & Anise & $\mathrm{H}$ & Annual & Fruits & Internal & Carminative and culinary use \\
\hline 79 & Pistachia atlantica Desf. & Anacardiacea & $\begin{array}{l}\text { Banak, Kalang } \\
\text { Kaleh, Kaleh } \\
\text { van }\end{array}$ & $\begin{array}{c}\text { Pesteh } \\
\text { Koohi/Baneh }\end{array}$ & Pistache & $\mathrm{T}$ & Perennial & Fruits, Resin & Internal & $\begin{array}{c}\text { Mind stranger, anti-hemorrhoid, } \\
\text { laxative, stomach stranger and bone } \\
\text { pain }\end{array}$ \\
\hline 80 & Pistachia khinjuk Stocks. & Anacardiacea & \begin{tabular}{|c|} 
Koleng Narmeh, \\
Narmeh van \\
\end{tabular} & Khenjuk & Pistache & $\mathrm{T}$ & Perennial & Fruits, Resin & Internal & $\begin{array}{l}\text { Digestive discords, diuretic, asthma, } \\
\text { stomach stranger and fragrant mouth }\end{array}$ \\
\hline 81 & Portulaca oleracea L. & Portulacaceae & $\begin{array}{c}\text { Perpelik, Denan } \\
\text { tizkarak }\end{array}$ & Khorfeh & Purslane & $\mathrm{H}$ & Annual & $\begin{array}{l}\text { Roots, Stem, } \\
\text { Leaves, Latex }\end{array}$ & Internal & Edible as vegetable, anti-parasite \\
\hline 82 & $\begin{array}{l}\text { Prangos ferulacea (L.) } \\
\text { Lindl. }\end{array}$ & Apiaceae & Bale har, Ginoo & Jooshir & Prangos & $\mathrm{H}$ & Perennial & Leaves, Flowers & $\begin{array}{c}\text { External/ } \\
\text { Internal } \\
\end{array}$ & Laxative \\
\hline 83 & $\begin{array}{c}\text { Prosopis farcta } \\
\text { (Banks \& Soland) Macbr. }\end{array}$ & Mimosaceae & \begin{tabular}{|c|}
$\begin{array}{c}\text { Belaveri, } \\
\text { Broweri, Khosh } \\
\text { khah shak }\end{array}$ \\
\end{tabular} & Kahoorak & $\begin{array}{l}\text { Syrian } \\
\text { mesquite }\end{array}$ & $\mathrm{S}$ & Perennial & Fruits & Internal & $\begin{array}{l}\text { Blood thinner and anti-diabetic } \\
\text { (Reduction of blood glucose) }\end{array}$ \\
\hline 84 & $\begin{array}{l}\text { Quercus brantii Lindl. var } \\
\text { persica (Jaub. \& Spach) } \\
\text { Zohary }\end{array}$ & Fagaceae & Bali, Bero & Baloot & Oak & $\mathrm{T}$ & Perennial & Fruits, Seed bark & Internal & $\begin{array}{l}\text { Gastric ulcer, stringent, sore throat } \\
\text { and anti-diabetes }\end{array}$ \\
\hline 85 & Rheum ribes L. & Polygonaceae & Ribas & Rivas & Rhubarb & $\mathrm{H}$ & Perennial & Stem & Internal & $\begin{array}{c}\text { Edible as vegetable, anti-hypertensive } \\
\text { and } \\
\text { decrease triglyceride }\end{array}$ \\
\hline 86 & Rhus coriaria L. & Anacardiaceae & Sumakh & Sumagh & Sicilian sumac & $\mathrm{S}$ & Perennial & Fruits & Internal & $\begin{array}{c}\text { Stomachic, anti-diarrheal, } \\
\text { tonic, digestive discords and culinary } \\
\text { use }\end{array}$ \\
\hline 87 & Rosa canina L. & Rosaceae & Goltieureg & $\begin{array}{l}\text { Nastaran-e- } \\
\text { vahshi }\end{array}$ & Dog rose & $\mathrm{S}$ & Perennial & Flowers & Internal & Indigestion \\
\hline 88 & Rosa damascena Mill. & Rosaceae & Gol bakhi & $\begin{array}{l}\text { Gol-e- } \\
\text { mohammadi }\end{array}$ & Persian rose & $\mathrm{S}$ & Perennial & Flowers & Internal & Indigestion \\
\hline 89 & $\begin{array}{l}\text { Rubus anatolicus (Focke.) } \\
\text { Focke ex Hausskn. }\end{array}$ & Rosaceae & Tiyarak & $\begin{array}{c}\text { Tameshk barg } \\
\text { narvani }\end{array}$ & $\begin{array}{l}\text { Elm-leaved } \\
\text { blackberry }\end{array}$ & $\mathrm{S}$ & Perennial & Fruits & Internal & Stomachic, anti-parasite \\
\hline 90 & $\begin{array}{c}\text { Rumex ephedroides } \\
\text { Bornm. }\end{array}$ & Polygonaceae & Torsheh mast & $\begin{array}{c}\text { Torshak-e- } \\
\text { rishbozi }\end{array}$ & Dock & $\mathrm{H}$ & Annual & Leaves & External & Anti-inflammatory \\
\hline 91 & Salix alba L. & Salicaceae & Vi, Bi & Bid-e-sephid & White willow & $\mathrm{T}$ & Perennial & Bark, Leaves & $\begin{array}{l}\text { Internal/ } \\
\text { External }\end{array}$ & Anti-fever \\
\hline 92 & Salsola vermiculata L. & Chenopodiaceae & Shoor & $\begin{array}{l}\text { Alaf shoor-e- } \\
\text { sharghi }\end{array}$ & Saltwort, R & $\mathrm{S}$ & Perennial & Stem, Leaves & Internal & Laxative, anti-ascorbic \\
\hline 93 & Salvia palaestina Benth. & Lamiaceae & Cherogi & $\begin{array}{c}\text { Maryam goli } \\
\text { falestini }\end{array}$ & Annual clary & $\mathrm{H}$ & Annual & $\begin{array}{c}\text { Leaves, } \\
\text { Inflorescence }\end{array}$ & Internal & $\begin{array}{c}\text { Women fertility and women } \\
\text { infections }\end{array}$ \\
\hline 94 & Scrophularia deserti Del. & Scrophulariaceae & Benjek mashin, & $\begin{array}{c}\text { Gol-e- } \\
\text { maymoni }\end{array}$ & Figwort & $\mathrm{H}$ & Perennial & Stem, Leaves & External & $\begin{array}{c}\text { Wound and burn healing } \\
\text { and antimicrobial }\end{array}$ \\
\hline 95 & Salvia sclarea L. & Lamiaceae & Maryam goli & Maryam goli & Clary & $\mathrm{H}$ & Perennial & $\begin{array}{l}\text { Leaves, Seed, } \\
\text { Inflorescence }\end{array}$ & Internal & Cold and anti-fever \\
\hline 96 & Satureja khuzistanica & Lamiaceae & Jatareh & Marzeh & Summer & $\mathrm{H}$ & Annual & Leaves, Stem, & Internal & Indigestion, headache, gastric pain, \\
\hline
\end{tabular}




\begin{tabular}{|c|c|c|c|c|c|c|c|c|c|c|}
\hline & Jamzad. & & & & savory & & & Flowers & & women infections, diuretic and spice \\
\hline 97 & $\begin{array}{c}\text { Scrophularia striata } \\
\text { Boiss. }\end{array}$ & Scrophulariaceae & Teshneh dari & $\begin{array}{c}\text { Gol-e- } \\
\text { maymoni }\end{array}$ & Figwort & $\mathrm{H}$ & Perennial & Stem, Leaves & External & $\begin{array}{c}\text { Wound and burn healing } \\
\text { and antimicrobial }\end{array}$ \\
\hline 98 & $\begin{array}{c}\text { Seidlitzia rosmarinus (Ehrh.) } \\
\text { Bge. }\end{array}$ & Chenopodiaceae & $\begin{array}{c}\text { Benjak shenan, } \\
\text { Hejhedan }\end{array}$ & Eshnan & Seidlitzia & $\mathrm{S}$ & Perennial & Leaves & External & $\begin{array}{l}\text { Used as washing, good hair condition } \\
\text { and plant shampoo }\end{array}$ \\
\hline 99 & Sesamum indicum L. & Pedaliaceae & Konjed & Konjed & Sesame & $\mathrm{H}$ & Annual & Seed & External & Burn healing \\
\hline 100 & Sinapis arvensis L. & Brassicaceae & Khartal, Terpeki & $\begin{array}{c}\text { Khardal-e- } \\
\text { zagrosi }\end{array}$ & Mustard & $\mathrm{H}$ & Annual & Stem, Flowers & Internal & Laxative and stomachic \\
\hline 101 & $\begin{array}{l}\text { Smyrnium cordifolium } \\
\text { Boiss. }\end{array}$ & Apaiacea & Pinomeh, Vangi & Avandol & Alexanders & $\mathrm{H}$ & Biannual & Stem & Internal & Indigestion and stomachic \\
\hline 102 & Solanum nigrum L. & Solanaceae & \begin{tabular}{|c|} 
Roarazak, \\
tamato kivi leh \\
\end{tabular} & $\begin{array}{c}\text { Sag angor, } \\
\text { Tajrizi-e-siyah } \\
\end{array}$ & $\begin{array}{c}\text { Black } \\
\text { nightshade }\end{array}$ & $\mathrm{H}$ & Annual & Fruits & External & $\begin{array}{c}\text { Skin diseases, wound healing } \\
\text { and eczema }\end{array}$ \\
\hline 103 & $\begin{array}{l}\text { Sorghum halepense (L.) } \\
\text { Pers. } \\
\end{array}$ & Poaceae & Helit & $\begin{array}{l}\text { Ghiagh, } \\
\text { Chaeer }\end{array}$ & Johnson grass & $\mathrm{H}$ & Perennial & $\begin{array}{l}\text { Leaves, Stem, } \\
\text { Flowers } \\
\end{array}$ & Internal & Abortion for human and animals \\
\hline 104 & $\begin{array}{l}\text { Stachys lavandulifolia } \\
\text { Vahl. }\end{array}$ & Lamiaceae & Colpar & Sonbolehei & Stachys & $\mathrm{H}$ & Perennial & Leaves, Flowers & Internal & $\begin{array}{c}\text { Carminative, rheumatism, indigestion, } \\
\text { headache, sedative, cardio tonic and } \\
\text { anti-anxiety }\end{array}$ \\
\hline 105 & Stipa capensis Thunb. & Poaceae & $\begin{array}{c}\text { Gol koo, } \\
\text { Bahmah giah }\end{array}$ & Chaman sozani & Needle grass & $\mathrm{H}$ & Annual & Flowers & Internal & $\begin{array}{l}\text { Nerve system problems } \\
\text { and gastric discords }\end{array}$ \\
\hline 106 & $\begin{array}{l}\text { Tamarix ramosissima } \\
\text { Ledeb. saltcedar }\end{array}$ & Tamaricaceae & Shoor gaz & Gaz-e-shahi & Tamarisk & $\mathrm{T}$ & Perennial & $\begin{array}{l}\text { Leaves and } \\
\text { Resin }\end{array}$ & $\begin{array}{l}\text { Internal/ } \\
\text { External } \\
\end{array}$ & $\begin{array}{l}\text { Dermal discords, wound healing and } \\
\text { sputum }\end{array}$ \\
\hline 107 & $\begin{array}{l}\text { Tanacetum polycephalum } \\
\text { Schultz. }\end{array}$ & Asteraceae & Samsa & $\begin{array}{l}\text { Minay-e- } \\
\text { porkopeh }\end{array}$ & Tansy & $\mathrm{H}$ & Perennial & Leaves & External & $\begin{array}{c}\text { Anti-hemorrhoid, } \\
\text { anti-inflammatory and sting }\end{array}$ \\
\hline 108 & Teucrium polium L. & Lamiaceae & Miyere nekhe & $\begin{array}{l}\text { Maryam } \\
\text { nokhodi }\end{array}$ & Germander & $\mathrm{H}$ & Perennial & Leaves, Flowers & Internal & $\begin{array}{l}\text { Anti-septic for gastric and fragrant } \\
\text { mouth }\end{array}$ \\
\hline 109 & Thymbra spicata L. & Lamiaceae & Azboh, Hazboh & $\begin{array}{l}\text { Avishan-e- } \\
\text { zophaye }\end{array}$ & Thyme & $\mathrm{H}$ & Perennial & $\begin{array}{l}\text { Leaves and } \\
\text { Inflorescence }\end{array}$ & Internal & $\begin{array}{l}\text { Spice, cough, antibacterial } \\
\text { and carminative }\end{array}$ \\
\hline 110 & $\begin{array}{l}\text { Tragopogon graminifolius } \\
\text { DC. }\end{array}$ & Asteraceae & Haplook & Shang & Salsify & $\mathrm{H}$ & Annual & Root, Flowers & $\begin{array}{l}\text { Internal/ } \\
\text { External }\end{array}$ & $\begin{array}{l}\text { Emollient, sore throat } \\
\text { and wound healing }\end{array}$ \\
\hline 111 & Trifolium repens L. & Fabaceae & She pareh & $\begin{array}{c}\text { Shabdar-e- } \\
\text { sephid }\end{array}$ & White clover & $\mathrm{H}$ & Biannual & $\begin{array}{l}\text { Leaves and } \\
\text { Inflorescence }\end{array}$ & $\begin{array}{l}\text { Internal/ } \\
\text { External }\end{array}$ & Analgesia and dermal discords \\
\hline 112 & Typha domingensis Pers. & Typhaceae & Lovan & Loei & Cat's tail & $\mathrm{H}$ & Perennial & Pollen, Rhizome & Internal & Anti-fever \\
\hline 113 & Ulmus glabra Hudson. & Ulmaceae & Vazam & $\begin{array}{c}\text { Narvan-e- } \\
\text { barg riz }\end{array}$ & Elm & $\mathrm{T}$ & Perennial & Leaves & Internal & $\begin{array}{c}\text { Heart discords } \\
\text { and fertility discords }\end{array}$ \\
\hline 114 & Verbascum alepense Benth. & Scrophulariaceae & Gol zardeh & Gol-e-mahour & Mullein & $\mathrm{H}$ & Perennial & $\begin{array}{l}\text { Leaves and } \\
\text { Flowers }\end{array}$ & $\begin{array}{l}\text { Internal/ } \\
\text { External } \\
\end{array}$ & $\begin{array}{l}\text { Anti-fever, dermal discords } \\
\text { and wound healing }\end{array}$ \\
\hline 115 & Vicia angustifolia L. & Fabaceae & Masheh maran & Mashak-e-barg & Narbonne & $\mathrm{H}$ & Annual & Fruits & Internal & Cough \\
\hline
\end{tabular}




\begin{tabular}{|c|c|c|c|c|c|c|c|c|c|c|}
\hline & & & & pahn & vetch & & & & & \\
\hline 116 & Viscum album L. & Loranthaceae & Darpechanak & Darvash & $\begin{array}{c}\text { White } \\
\text { mistletoe }\end{array}$ & $\mathrm{T}$ & Perennial & $\begin{array}{l}\text { Leaves, Stem, } \\
\text { Flowers } \\
\end{array}$ & $\begin{array}{c}\text { External/ } \\
\text { Internal }\end{array}$ & $\begin{array}{c}\text { Body pain, knew joint pain } \\
\text { and abscess }\end{array}$ \\
\hline 117 & $\begin{array}{l}\text { Vitex pseudo-negundo } \\
\text { (Hausskn.) Hand-mzt. }\end{array}$ & Verbenaceae & Keref, Kerof & Bangaro & Chaste tree & $\mathrm{S}$ & Perennial & Leaves & Internal & Increased milk \\
\hline 118 & Xanthium spinosum L. & Asteraceae & Chazanak & Zardineh & Cocklebur & $\mathrm{H}$ & Annual & $\begin{array}{l}\text { Leaves and } \\
\text { Fruits }\end{array}$ & External & Dermal discords, diuretic \\
\hline 119 & Xanthium strumarium L. & Asteraceae & Chazanak & Zardineh & Cocklebur & $\mathrm{H}$ & Annual & $\begin{array}{l}\text { Leaves and } \\
\text { Fruits }\end{array}$ & Internal & Laxative, stomach, tonic \\
\hline 120 & Ziziphora capitata L. & Lamiaceae & Kakooti & $\begin{array}{c}\text { Moshk } \\
\text { taramoshk }\end{array}$ & Ziziphora & $\mathrm{H}$ & Annual & $\begin{array}{l}\text { Leaves and } \\
\text { Inflorescence }\end{array}$ & Internal & Spice and culinary \\
\hline 121 & $\begin{array}{l}\text { Ziziphus nummularia } \\
\text { (Burm. F.) Wighth \& Arn. }\end{array}$ & Ramnaceae & Darak, Konar & Ramlik & Camel thorn & $\mathrm{T}$ & Perennial & $\begin{array}{l}\text { Leaves and } \\
\text { Fruits }\end{array}$ & External & Gastric pain and stomachic \\
\hline 122 & $\begin{array}{l}\text { Ziziphus spina-christi (L.) } \\
\text { Willd. }\end{array}$ & Rhamnaceae & Sedr, Azakonar & Konar & Christ's thorn & $\mathrm{T}$ & Perennial & $\begin{array}{l}\text { Leaves and } \\
\text { Fruits }\end{array}$ & External & Anti-dandruff and anti-hair loss \\
\hline
\end{tabular}

Habit: T: Tree, S: Shrub, H: Herb 
Table 2: Major ailments treated by the local inhabitants of the Dehloran and Abdanan regions, Ilam Province, Iran using medicinal plants species

\begin{tabular}{|c|c|c|c|}
\hline S. no. & Ailments & No. of plants & Percentage \\
\hline 1 & Gastric pain & 17 & 5.69 \\
\hline 2 & Anti-septic & 14 & 4.68 \\
\hline 3 & Indigestion & 14 & 4.68 \\
\hline 4 & Laxative & 14 & 4.68 \\
\hline 5 & Wound Healing & 14 & 4.68 \\
\hline 6 & Anti-calculus & 10 & 3.34 \\
\hline 7 & Stomachic & 10 & 3.34 \\
\hline 8 & Cough & 9 & 3.01 \\
\hline 9 & Sore throat & 9 & 3.01 \\
\hline 10 & Anti-parasite & 8 & 2.68 \\
\hline 11 & Dermal discords & 8 & 2.68 \\
\hline 12 & Tonic & 8 & 2.68 \\
\hline 13 & Anti-fever & 7 & 2.34 \\
\hline 14 & Carminative & 7 & 2.34 \\
\hline 15 & Cold & 6 & 2.01 \\
\hline 16 & Digestive & 6 & 2.01 \\
\hline 17 & Emollient & 6 & 2.01 \\
\hline 18 & Headache & 6 & 2.01 \\
\hline 19 & Kidney pain & 6 & 2.01 \\
\hline
\end{tabular}




\begin{tabular}{|c|c|c|c|}
\hline 20 & Regulation Blood sugar & 6 & 2.01 \\
\hline 21 & Sedative & 6 & 2.01 \\
\hline 22 & Burn healing & 5 & 1.67 \\
\hline 23 & Diuretic & 5 & 1.67 \\
\hline 24 & Hair treatment & 5 & 1.67 \\
\hline 25 & Rheumatism & 5 & 1.67 \\
\hline 26 & Anti-diarrheal & 4 & 1.34 \\
\hline 27 & Anti-Inflammatory & 4 & 1.34 \\
\hline 28 & Eczema & 4 & 1.34 \\
\hline 29 & Heart tonic & 4 & 1.34 \\
\hline 30 & Nerve System Discords & 4 & 1.34 \\
\hline 31 & Toothache & 4 & 1.34 \\
\hline 32 & Anti-microbial & 3 & 1.00 \\
\hline 33 & Anti-wart & 3 & 1.00 \\
\hline 34 & Stomach stranger & 3 & 1.00 \\
\hline 35 & Ethno-Veterinary & 3 & 1.00 \\
\hline 36 & Abortion & 2 & 0.67 \\
\hline 37 & Analgesia & 2 & 0.67 \\
\hline 38 & Anti-hemorrhoid & 2 & 0.67 \\
\hline 39 & Anti-hypertensive & 2 & 0.67 \\
\hline 40 & Body pain & 2 & 0.67 \\
\hline 41 & Fragrant mouth & 2 & 0.67 \\
\hline 42 & Soporific & 2 & 0.67 \\
\hline
\end{tabular}




\begin{tabular}{|c|c|c|c|}
\hline 43 & Women infections & 2 & 0.67 \\
\hline 44 & Abscess & 1 & 0.33 \\
\hline 45 & Anti-anxiety & 1 & 0.33 \\
\hline 46 & Anti-ascorbic & 1 & 0.33 \\
\hline 47 & Anti-bacterial & 1 & 0.33 \\
\hline 48 & Anti-dandruff & 1 & 0.33 \\
\hline 49 & Anti-hair loss & 1 & 0.33 \\
\hline 50 & Anti-leech & 1 & 0.33 \\
\hline 51 & Anti-Tumor & 1 & 0.33 \\
\hline 52 & Anti-urticarial & 1 & 0.33 \\
\hline 53 & Appetizer & 1 & 0.33 \\
\hline 54 & Blood thinner & 1 & 0.33 \\
\hline 55 & Bone pain & 1 & 0.33 \\
\hline 56 & Bronchits & 1 & 0.33 \\
\hline 57 & Child ear pain & 1 & 0.33 \\
\hline 58 & Decrease triglyceride & 1 & 0.33 \\
\hline 59 & Duodenal pain & 1 & 0.33 \\
\hline 60 & Eyes discords & 1 & 0.33 \\
\hline 61 & Fattening & 1 & 0.33 \\
\hline 62 & Fertility discords & 1 & 0.33 \\
\hline 63 & Heart discords & 1 & 0.33 \\
\hline 64 & Hemagglutinate & 1 & 0.33 \\
\hline 65 & Hepatoprotective & 1 & 0.33 \\
\hline
\end{tabular}




\begin{tabular}{|c|c|c|c|}
\hline 66 & Increased milk & 1 & 0.33 \\
\hline 67 & Intestinal Problem & 1 & 0.33 \\
\hline 68 & Joundice & 1 & 0.33 \\
\hline 69 & Knew joint pain & 1 & 0.33 \\
\hline 70 & Menorrhagia & 1 & 0.33 \\
\hline 71 & Mind stranger & 1 & 0.33 \\
\hline 72 & sputum & 1 & 0.33 \\
\hline 73 & Reduced Cholesterol & 1 & 0.33 \\
\hline 74 & Sciatica & 1 & 0.33 \\
\hline 75 & Scorpion bite & 1 & 0.33 \\
\hline 76 & Snake bit & 1 & 0.33 \\
\hline 77 & Sting & 1 & 0.33 \\
\hline 78 & Urine Tube & 1 & 0.33 \\
\hline 79 & Women Period disords & 1 & 0.33 \\
\hline
\end{tabular}


Culinary and spice uses

At present, 14 plants are collected in the study area for their use in culinary, spice and food. Among them, 10 species are reported also for therapeutic use (see Table 1); 3 are used only as food. The villagers employ Crocus sativus, Cerasus mahaleb, Ferulago angulata, Mentha longifolia, Pimpinella anisum, Satureja khuzistanica, Thymbra spicata, Ziziphora capitata and Rhus coriaria as flavoring agents. The leaves and stem of Mentha longifolia, Portulaca oleracea, Rheum ribes, Allium ampeloprasum subsp. iranicum and bulbs of Echinops viscidulus are used as wild vegetables. The fruits of Crataegus azarolus, Cerasus mahaleb, Ficus carica, Pistachia atlantica, Pistachia khinjuk, Prosopis farcta, Rubus anatolicus, Ziziphus nummularia, Ziziphus spina-christi, Amygdalus arabica, Amygdalus lycioides and Elaeagnus angustifolia are used as wild fruit. Astaragalus glaucacanthus are used as culinary and confectionery, tonic, gastric pain, headache and or as wild fruit. They boil tender leaves of Cardaria draba to prepare soups and Gundelia tournefortii to prepare pickled.

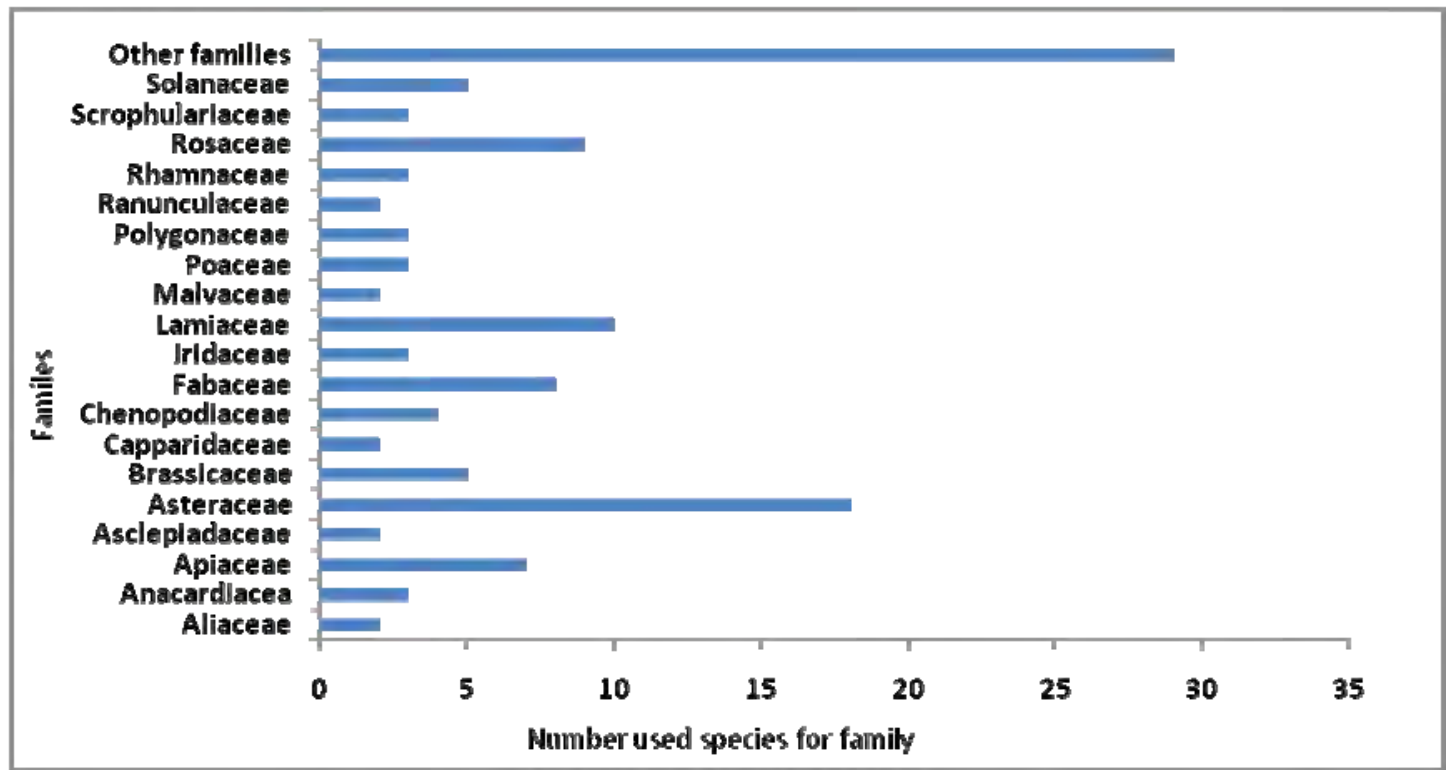

Figure 1: Frequency of plant families used in Dehloran and Abdanan district, Ilam province, Iran

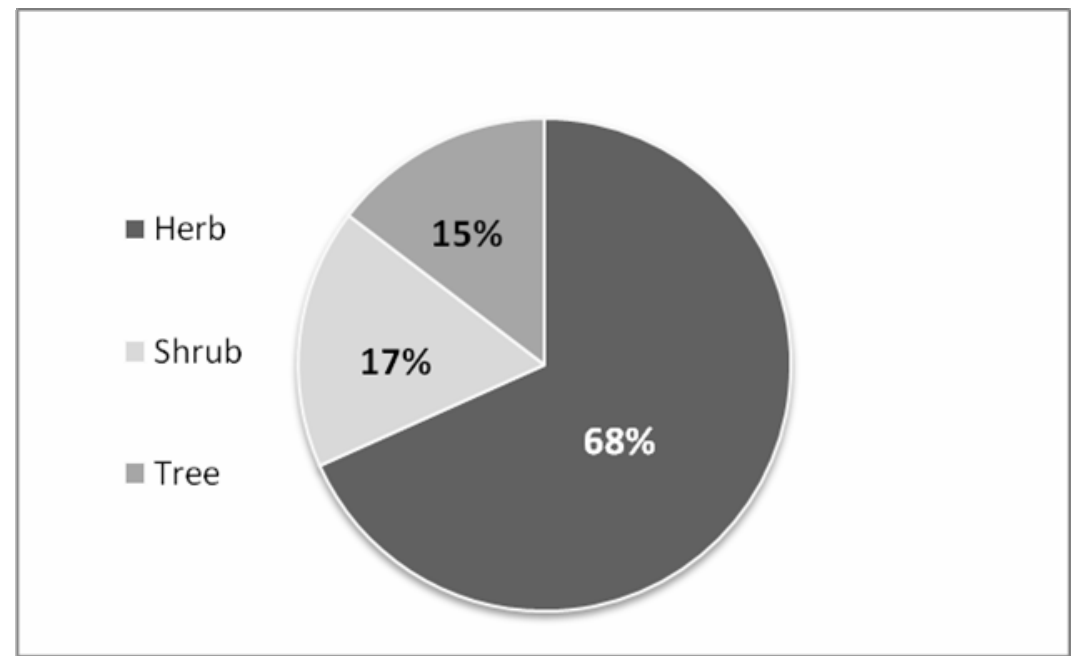

Figure 2: Frequency of habit plants used in Dehloran and Abdanan district, Ilam province, Iran 


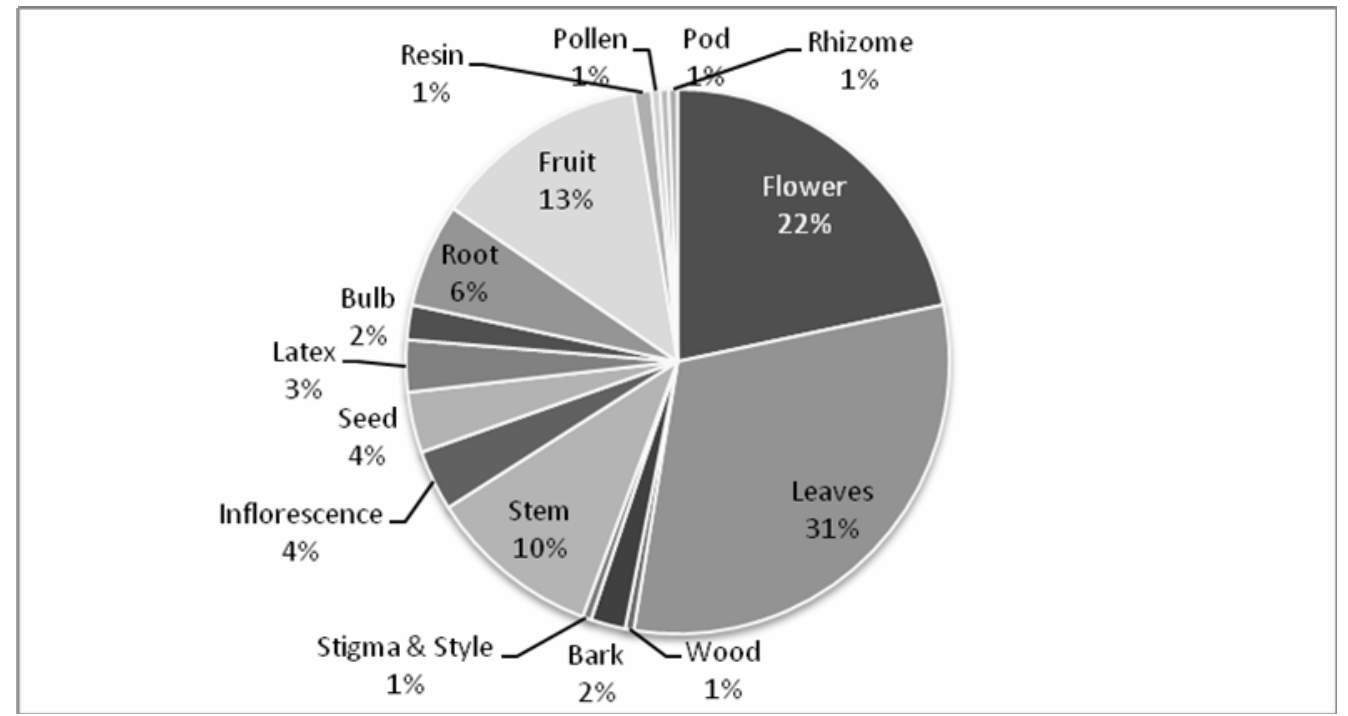

Figure 3: Frequency of plant parts used in Dehloran and Abdanan district, Ilam province, Iran

\section{Ailments treated}

The 122 medicinal plant species were used in treating 79 different types of ailments (Table 2). The highest number of plant species (17 species) was used for the treatment of gastric disorders followed by antiseptic, indigestion, laxative and wound healing (14 species).

\section{Veterinary uses}

Interviewees have mentioned few species for treatment of animals. Nicotina tabacum (reported by the $10 \%$ of informants) is only used in veterinary medicine, as anti-parasitic (Anti-leech for example: Limnatis nilotica) and antifungal (antidermatophytosis) for external use. Sorghum halepense and Amygdalus arabica, used commonly for humans (see Table 1), are employed as remedies for domestic animals. The leaf and stem of Sorghum halepense is administered externally to animals for abortion. The oil seed of Amygdalus arabica is used externally for body pain.

\section{Marketability of medicinal plants}

Nicotiana tabacum used as a stimulant (smoking), Cannabis sativa is used as a nut, while Sesamum indicum is used as a culinary agent and nut (oil crop) and Crocus sativus is used as a spice and culinary (color and flavor of rice and other foods) medicinal plants were sold in the market for their respective indications.

\section{Discussions and conclusions}

In present study, we have compared our ethnobotanical data with the data present in Iranian Medicinal plant literatures (Afshar, 1990; Amin, 1991; Ayiineh Chii, 1989; Ghasemi Pirbalouti, 2009a,b; Ghorbani, 2005; Hovayzeh et al., 2001; Miraldi et al., 2001; Mir-Heidari, 1993; Rojhan, 1991; Salehi Surmaghi et al., 1992; Zargari, 1989-1992). Most of the plants indicated by the interviewees are reported in Iranian literature, but not in every occasion were the actions attributed to a plant the same. For example, there are not reports in the official Iranian phytotherapy of the use of Atriplex leucoclada and Echinops viscidulus as an emollient, cough and sore throat; Avena wiestii as treatment of gastric pain and rheumatism; Centaurea iberica, Centaurea ovina, Centaurea intricate and Picnomon acarna as treatment of gastric pain; Cerasus microcarpa subsp. microcarpa as sedative, anticalculus and anti-fever; Cirsium congestum and Crocus haussknechtii as anti-septic for gastric; Colchicum kotschyi as treatment of rheumatism; Consolida orientalis as laxative and anti-parasite, Ephedra ciliata as anti-bacterial and anti-fever, Euphorbia macroclada as treatment of wart; Lonicera nummulariifolia as anti-fever, anti-diarrheal and sedative; Nepeta persica as carminative and anti-urticarial; Noaea mucronata and Onobrychis elymaitica as anti-calculus and kidney problems; Opoponex hispidus as antiseptic; Prangos ferulacea as laxative; Periploca aphylla as anti-inflammatory; Prosopis farcta as blood thinner and anti-diabetic (reduction of blood glucose); Salvia palaestina as women fertility and women infections; Satureja khuzistanica as indigestion; headache, women infections and diuretic; Scrophularia deserti and Scrophularia striata as wound and burn 
healing; Stipa capensis as treatment of nerve system problems and gastric discords; Tamarix ramosissima as treatment of dermal discords, wound healing and sputum ; Thymbra spicata as treatment of cough, antibacterial and carminative; Ulmus glabra as treatment of heart discords and fertility discords; Verbascum alepense as anti-fever, dermal discords and wound healing; Vitex pseudo-negundo as increased milk; Nicotiana tabacum as treatment animal (Anti-leech and anti-dermatophytosis).

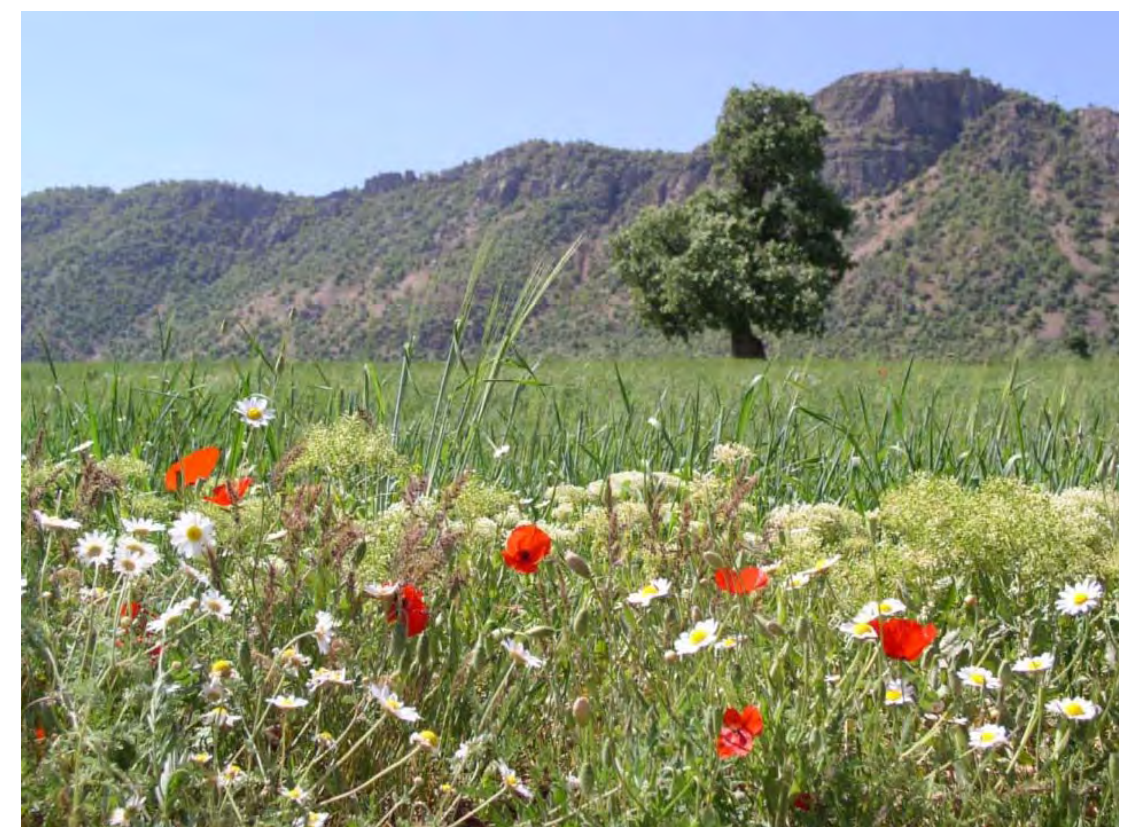

Figure 4: Landscape of cover plants on rangelands in Ilam province, Iran

Our study contributed confirmed the ethnobotanical knowledge of Abdanan and Dehloran districts, filling a long overlooked gap. It once more remarked the relationship existing between plant diversity and the degree of ethnobotanical knowledge recorded. The former has been retained thanks to a long history of nature preservation in the study area. It is worth highlighting that we found some young people who still retain ethnobotanical knowledge or at least express interest towards traditional uses, so that they performed well as key informants. This clearly derives from the cultural and professional opportunities offered by living in a famous protected area where nature is still an important issue for local communities. However, even under these circumstances many uses have disappeared and some forgotten by otherwise experienced informants. We believe that cultural diversity should be seen in a broader sense as part of biodiversity of a region, especially where disentangling human influence and nature is virtually impossible. Traditional knowledge should therefore feature more often in the agendas of nature reserves besides biological richness as a value to preserve for the future. In general, the people of the study area still have a strong belief in the efficiency and success of medicinal plants. The results of our study reveal that some of the plant species do play an important role in the primary healthcare system of this tribal community.

\section{Acknowledgment}

The authors are thanking the tribal people for providing information on the medicinal uses of plants.
Appendix 1
1. Date
2. Village
3. Informant name and surname
4. Age
5. Degree of education
6. Family origins
7. Which wild plants do you use to treat the different ailments?

8. How you have learned to recognize them?

9. Which plant parts do you use?

10. What is the vernacular name of these plants/part plants?

11. Can you describe the preparation of remedy in detail?

12. When should the medicine be taken and for how long?

13. Internal or external administration?

14. Where does this knowledge arrive from? 


\section{References}

1. Afshar, I. (1990). The Iranians Traditional Medicine. Homa Press, Tehran, Iran.

2. Amin, G. (1991). Popular Medicinal Plants of Iran. Iranian Research Institute of Medicinal Plants, Tehran, Iran.

3. Ayiineh Chii, Y. (1989). Medicinal Plants and Materia Medica. University Publication, Tehran, Iran.

4. Ghahreman, A. (1987-1989). Flora of Iran. Department of Botany, Institute of Forest Sciences, Tehran, Iran.

5. Ghasemi Pirbalouti, A. (2009a). Medicinal plants used in Chaharmahal and Bakhtyari districts, Iran. Herba Polonica, 55: 69-75.

6. Ghasemi Pirbalouti, A. (2009b). Iranian Medicinal and Aromatic Plants. Islamic Azad University Press, Shahrekord, Iran.

7. Ghorbani, A. (2005). Studies on pharmaceutical ethnobotany in the region of Turkmen Sahra, north of Iran. J Ethnopharmacol, 102: 5868.

8. Hovay $12-16$.

9. Ilam Meteorology Office. (2010). Climate and Geography of Ilam Province. Available on Internet at: http://www.weather.ir

10. Management and Planning Organization of Ilam (MPOI). (2003). Study of Survey Development Capability of Ilam, Flora Division. Management and Planning Organization of Ilam Press.

11. Miraldi, E., Ferri, S. and Mostaghimi, V. (2001). Botanical drugs and preparations in the traditional medicine of west Azerbaijan (Iran). J Ethnopharmacol, 75: 77-87.

12. Mir-Heidari, H. (1993). Encyclopedia of Medicinal Plants of Iran. Islamic Culture Press, Tehran, Iran.

13. Mozaffarian, V. (1996). Encyclopedia of Iranian Plants. Farhang Moaser Publication, Tehran, Iran.

14. Mozaffarian, V. (2008). Flora of Ilam. Farhang Moaser Publication, Tehran, Iran.

15. Rechinger, K. H. (1963-1998). Flora Iranica. Vol. 1-173. Akademische Druck und Verlagsanstalt. Graz, Austria

16. Rojhan, M. S. (1991). Herbal Drugs and Treatment with Medicinal Plants. Marshal Press, Isfahan, Iran.

17. Salehi Surmaghi, M. H., Aynehchi, Y., Amin, G. and Mahmoodi, Z. (1992). Survey of Iranian plants for saponins, alkaloids, flavonoids and tannins. Daru, 2: 281-291.

18. Zargari, A. (1989-1992). Medicinal Plants. Vol. 1-6. University Publication, Tehran, Iran. 